\title{
FLUTUAÇÃO POPULACIONAL E DEPENDÊNCIA ESPACIAL DE Aleurocanthus woglumi ASHBY, 1915 (HEMIPTERA: ALEYRODIDAE) EM Citrus latifolia ${ }^{1}$
}

\author{
DANIELE LAVRA VIEIRA², ANGELO LUIZ TADEU OTTATI ${ }^{3}$, \\ RAIMUNDA NONATA SANTOS DE LEMOS 4 , \\ GISLANE DA SILVA LOPES ${ }^{5}$, JOSÉ RIBAMAR GUSMÃO ARAUJO ${ }^{6}$
}

RESUMO-A mosca-negra-dos-citros, Aleurocanthus woglumi, apresenta elevada capacidade de dispersão e adaptação a diversas condições climáticas, além de grande potencial reprodutivo. O presente trabalho teve como objetivo avaliar a flutuação populacional e caracterizar o padrão de dependência espacial de $A$. woglumi em um pomar de lima- ácida Tahiti (Citrus latifolia, Tanaka), no município de São José de Ribamar, Estado do Maranhão. Um sistema de posicionamento global (GPS) e um sistema de informação geográfica (SIG) foram usados para localizar os pontos das amostras, caracterizar o padrão de distribuição, calcular a área de agregação e elaborar mapas de distribuição dos adultos de mosca-negra-dos-citros dentro do pomar. O número médio total de moscas negras foi maior durante a estação chuvosa e observaram-se correlações significativas entre as variáveis precipitação pluvial, umidade relativa do ar e número total de adultos. A distribuição espacial $A$. woglumi no pomar é agregada, ajustando-se os variogramas calculados ao modelo esférico nas estações seca e chuvosa. Os insetos mostraram uma área de agregação média de $162.092 \mathrm{~m}^{2}$ na estação chuvosa e de $9.615 \mathrm{~m}^{2}$ na estação seca. Para se obter uma estimativa confiável de populações de mosca-negra-dos-citros, pelo menos uma armadilha deve ser usada a cada 17 hectares na estação chuvosa e uma armadilha por hectare na estação seca.

Termos pra indexação: Mosca-negra-dos-citros, distribuição espacial, geoestatística.

\section{POPULATION FLUCTUATION AND SPATIAL DEPENDENCE OF Aleurocanthus woglumi ASHBY, 1915 (HEMIPTERA: ALEYRODIDAE) ON Citrus latifolia}

\begin{abstract}
The citrus blackfly, Aleurocanthus woglumi, presents high dispersal ability and adaptability to diverse climatic conditions as well as large reproductive potential. This study aimed to evaluate the populational fluctuation and to characterize the pattern of spatial dependence of citrus blackfly in a commercial orchard of Tahiti lime (Citrus latifolia Tanaka), in São José de Ribamar county, state of Maranhão. A global positioning system (GPS) and geographic information system (GIS) were used to locate the points of the samples, to characterize the pattern of distribution, calculate the aggregation area and produce distribution maps of the adults of citrus blackfly in the orchard. The average total number of citrus blackflies was higher during the rainy season and there were significant correlations between the variables rainfall, relative humidity and total number of adults. The spatial distribution of $A$. woglumi in the orchard is aggregated, adjusting the calculated variograms to the spherical model in the dry and rainy seasons. The insects showed an area of mean aggregation of $162,092 \mathrm{~m}^{2}$ in the rainy season and $9,615 \mathrm{~m}^{2}$ in the dry season. Thus, to obtain a reliable estimate of citrus blackfly populations, at least one trap should be used every 17 hectares in the rainy season and one trap per hectare in the dry season.
\end{abstract}

Index terms: Citrus blackfly, spatial distribution, geostatistics.

\footnotetext{
${ }^{1}$ Trabalho 415-13. Recebido em: 17-10-2013. Aceito para publicação em: 29-09-2014. Parte da dissertação do primeiro autor apresentada ao Programa de Pós-Graduação em Agroecologia/UEMA.

${ }^{2,5}$ Engenheira Agrônoma, Mestre em Agroecologia Pelo Programa de Pós-Graduação em Agroecologia/CCA/UEMA, E-mails: d.lavra@ hotmail.com; gslopes11@yahoo.com

${ }^{3}$ Engenheiro Agrônomo, DSc. Fiscal Federal do MAPA-SEDESA/DT/SFA/MA. E-mail: angelo.ottati@agricultura.gov.br

${ }^{4,6}$ Engenheiro Agrônomo, DSc. Professor Adjunto do Depto. de Fitotecnia e Fitossanidade da Universidade Estadual do Maranhão

- UEMA; Caixa Postal 9, CEP 65055098, São Luís-MA. Email: r.lemos@elo.com.br (autor correspondente); gusmão@elo.com.br
} 


\section{INTRODUÇÃO}

O Brasil é um dos três maiores produtores mundiais de frutas, e a estimativa da produção de laranja em 2014 alcança 16,4 milhões de toneladas ou 402,5 milhões de caixas, aumento de $0,8 \%$ em relação a 2013. São Paulo é o principal produtor do País, respondendo por 72,0\% da produção (IBGE, 2014).

Apesar do cenário favorável, esse setor vem sendo ameaçado por uma praga quarentenária presente, Aleurocanthus woglumi Ashby, 1915 (Hemiptera: Sternorrhyncha: Aleyrodidae), que causa perdas de $20 \%$ a $80 \%$ em plantas cítricas pela sucção contínua de nutrientes e pelo aparecimento da fumagina sobre as folhas, dificultando o processo de respiração e fotossíntese das plantas, além de restringir o comércio com outras regiões livres da praga (RAGA; COSTA, 2008; ADEAL, 2010; MAPA, 2014).

Outro aspecto relevante consiste no fato de essa praga possuir hábito polífago, uma vez que, além de diversas espécies frutíferas, pode infestar plantas ornamentais e plantas daninhas, sendo facilmente transportada para outras regiões (RAGA; COSTA, 2008). É justamente sua capacidade de adaptação e dispersão em condições brasileiras e à falta de pesquisas e informações sobre essa praga, que a tornam tão preocupante aos fruticultores. Inicialmente encontrada no Estado do Pará, em 2001, disseminou-se para os Estados do Amapá, Amazonas, Tocantins, Maranhão e São Paulo (LEMOS et al., 2006; RONCHI-TELLES et al., 2009), Paraíba (LOPES et al., 2009), Bahia (SILVA et al., 2010), Ceará (ADAGRI, 2010), Rio Grande do Norte (ADEAL, 2010), Roraima (CORREIA et al., 2011), Pernambuco (MONTEIRO, 2012), Tocantins e Alagoas (MAPA, 2014).

Dentre as pesquisas realizadas com esse aleirodídeo, destacam-se os aspectos biológicos e os testes de preferência para oviposição. Ronchi-Telles et al. (2009) caracterizaram A. woglumi como uma espécie multivoltina, com ciclo médio de vida de $71,76 \pm 2,07$ dias. Lopes et al. (2013) observaram que este inseto apresenta preferência por ovipositar nas espécies cítricas (limoeiro, laranjeira e tangerineira), mantendo um padrão de não preferência em cajueiro e goiabeira, sendo que os hospedeiros laranjeira e mangueira não interferiram no ciclo biológico desta praga, com duração de 66,71 \pm 0,71 e 69,00 $\pm 0,65$ dias, respectivamente.

Todavia, faltam informações básicas para implementação de programas de manejo integrado de pragas, que só poderão ser alcançadas mediante pesquisas de monitoramento e amostragem corretas da praga no ambiente.

Segundo Blackshaw e Vernon (2006), o conhecimento da dinâmica espaço-temporal dos insetos em ecossistemas agrícolas é importante para o desenvolvimento de estratégias de manejo e redução do uso de pesticidas. Dessa forma, alguns estudos têm sido desenvolvidos com hemípteros em condições brasileiras, como os trabalhos realizados por Maruyama et al. (2006) e Ferreira Filho et al. (2008), que visaram a esclarecer a distribuição e a estrutura populacional de Oncometopia facialis Stal, 1869 (Hemiptera: Cicadellidae) e Glycaspis brimblecombei Moore, 1964 (Hemiptera: Psyllidae), respectivamente.

Pesquisas sobre monitoramento e uso de tecnologias de informações geográficas foram desenvolvidas com A. woglumi no Estado do Texas (FLETCHER et al., 2004; MEAGHER et al., 1991). Contudo, em condições brasileiras, esses estudos ainda são incipientes. Medeiros et al. (2009) avaliaram a dinâmica populacional da referida praga, mas é necessário determinar a distribuição espacial e construir um plano confiável de amostragem que permita estimar a densidade populacional e seu dano, o que permitirá tomar a decisão de controle, com consequente redução do custo de produção.

As relações espaciais dos insetos são, frequentemente, manifestações de características biológicas inerentes às necessidades espaciais das espécies, influenciadas pela planta hospedeira e pelo ambiente (TAYLOR, 1984), enquanto a dependência espacial é a característica decorrente do fato de que amostras espacialmente próximas são comumente mais semelhantes umas às outras do que amostras mais distantes (MATOS, 2000).

Normalmente, os métodos estatísticos convencionais descrevem a distribuição de uma população de insetos como agregada, uniforme ou aleatória, e segundo Farias et al. (2002), a distância entre os pontos amostrados não é levada em consideração. A geoestatística tem sido o método mais utilizado para estudar as populações. Permite detectar a variabilidade espacial e uma medida de correlação entre as amostras coletadas em campo por meio do semivariograma, que nada mais é do que o gráfico da semivariância estatística $(\gamma)$ em função da distância (h) entre os pares de pontos amostrados (TEIXEIRA, 2013).

Partindo-se do pressuposto de que o mapeamento permite a visualização espacial da praga no agroecossistema, possibilitando seu controle racional com aplicações direcionadas, redução nos custos de produção e diminuição dos impactos causados por produtos fitossanitários, este trabalho 
teve como objetivos avaliar a flutuação populacional e a dependência espacial da mosca-negra em um pomar de lima-ácida Tahiti (Citrus latifolia, Tanaka), visando a determinar o número de armadilhas necessárias para uma amostragem representativa de A. woglumi.

\section{MATERIAL E MÉTODOS}

O experimento foi instalado em pomar de lima-ácida Tahiti (C. latifolia) localizado no município de São José de Ribamar (MA), com área de 3,5 ha, espaçamento $7 \times 7 \mathrm{~m}$, com cinco anos de idade e coordenadas geográficas $02^{\circ} 33^{\prime}$ 04,8" $\mathrm{S}$ e $44^{0} 13^{\prime} 07,6$ " W. Durante todo o período de estiagem (agosto a dezembro), as plantas foram irrigadas pelo método de microaspersão e receberam os tratos culturais recomendados para a cultura, inclusive poda de limpeza e aplicação do ingrediente ativo imidacloprido, três meses antes do início do experimento.

As amostragens foram quinzenais, no período de janeiro/2007 a janeiro/2008. Para cada coleta, utilizaram-se 64 armadilhas amarelas adesivas (10 $\mathrm{cm}$ de largura e $21 \mathrm{~cm}$ de altura), espaçadas nas linhas e entrelinhas de plantio em $21 \mathrm{~m}$ e suspensas nas árvores a uma altura de 1,5m do solo, sempre dispostas na face norte.

Quanto à determinação da dependência espacial, os pontos amostrados foram georreferenciados com GPS, utilizando-se das coordenadas planas do Sistema Universal Transversal de Mercator (UTM) para sua localização.

As armadilhas foram substituídas a cada 15 dias e, para avaliação em laboratório, foram protegidas por filme de PVC em toda a face antes do transporte.

No Laboratório de Entomologia da Universidade Estadual do Maranhão (UEMA), os insetos capturados foram separados por sexo e contados com auxílio de um estereomicroscópio. Quando o número de insetos coletados foi muito grande, a armadilha era dividida em quadrantes, contando-se a quantidade presente em um dos quadrantes e multiplicando-se o valor obtido por quatro, totalizando-se o número de machos e fêmeas (NGUYEN et al., 1983).

Avaliou-se a flutuação populacional de machos, fêmeas e total de adultos coletados nas estações chuvosa e seca. Os dados diários de temperatura $\left({ }^{\circ} \mathrm{C}\right)$, umidade relativa do ar (\%) e precipitação pluvial ( $\mathrm{mm}$ ) foram obtidos junto ao Laboratório de Meteorologia do Núcleo Geoambiental da UEMA, a partir de uma plataforma de dados meteorológicos instalada na cidade de São Luís-MA, com coordenadas geográficas $44^{0} 12$ ' 42,7 " O e $02^{\circ} 35^{\prime} 34,6$ " S com 4,7 km de distância do pomar.

Para a correlação entre as variáveis climáticas e as curvas de flutuação populacional de adultos, utilizaram-se médias mensais de temperatura, umidade relativa do ar e o somatório da precipitação pluvial acumulada em períodos de 14 dias, imediatamente anteriores às coletas (Figura 1).

Realizou-se análise de correlação simples entre o número de insetos (machos, fêmeas e total) e as variáveis climáticas. Pelo teste de Shapiro-Wilk, observou-se que, para as variáveis analisadas, os dados não apresentavam distribuição normalizada, e que, nesse caso, o teste de Spearman seria o mais adequado (procedimento Univariate, $\mathrm{SAS}^{\circledR}, 2001$ ). Os dados foram transformados em $\sqrt{\mathrm{x}+0,5}$, e os coeficientes de correlação (r) foram testados pelo teste t de Student, ao nível de 5\% de probabilidade.

Os variogramas construídos foram adequados de acordo com o modelo que forneceu o melhor coeficiente de determinação (R2). Esse modelo foi validado pela relação $\mathrm{C} 0 / \mathrm{C} 0+\mathrm{C} 1$, que determina o índice $\mathrm{k}$, sendo que valores inferiores a 0,80 denotam a distribuição agregada às populações em estudo. $\mathrm{O}$ valor da semivariância no intercepto do eixo $\gamma(\mathrm{h})$ é chamado de efeito pepita (C ), por definição $\gamma(0)=0$ (zero). Entretanto, na prática, à medida que $\mathrm{h}$ tende para zero, $\gamma(\mathrm{h})$ aproxima-se de um valor positivo chamado efeito pepita (C), que representa a variabilidade em distâncias menores do que a distância da amostragem mínima realizada. Variação espacial (C1) é a diferença entre o patamar (C) e o efeito pepita (C0) (FARIAS et al., 2002).

As variâncias amostrais foram definidas em termos de $\gamma$ como uma função da distância de separação entre os pontos (h): $\gamma(\mathrm{h})=1 / 2 \mathrm{n}(\mathrm{h}) \Sigma$ $\left[\mathrm{Z}\left(\mathrm{x}_{\mathrm{i}}\right)-\mathrm{Z}\left(\mathrm{x}_{\mathrm{i}+\mathrm{h}}\right)\right]^{2}$, em que: $\mathrm{Z}\left(\mathrm{x}_{\mathrm{i}}\right)$ é o valor amostral medido ou contado no local $x_{i} ; Z\left(x_{i+h}\right)$ é o valor amostral medido ou contado no local $\mathrm{x}_{\mathrm{i}+\mathrm{h}}$ e $\mathrm{n}(\mathrm{h})$ é o número total de pares amostrais para cada uma das distâncias de separação (MATHERON, 1963).

A determinação da distância entre as armadilhas foi feita para uma direção geral, isto é, adotando-se um ângulo de tolerância de $90^{\circ}$. Isto significa que as distâncias entre os locais das armadilhas foram determinadas em todas as direções dentro da área amostrada.

As estimativas de valores em pontos não amostrados a partir de amostras coletadas, considerando a estrutura de dependência espacial do fenômeno, são um método de inferência espacial chamado de krigagem. E essa estimativa através 
do método da krigagem foi obtida pela seguinte expressão: $z^{*}\left(x_{0}\right)=\sum_{i=1}^{N} \lambda_{i} z\left(x_{i}\right)$, em que: $\mathrm{N}$ é o número de valores medidos $\mathrm{z}(\mathrm{x} i)$ envolvidos na estimativa; $\lambda i$ são as unidades associadas com cada valor medido (MATHERON, 1963).

As análises dos variogramas foram realizadas com o programa GS+. Os modelos foram adequados aos variogramas, e os dados foram krigados, e as estimativas krigadas foram utilizadas para produzir mapas de densidade da população de adultos da mosca-negra.

\section{RESULTADOS E DISCUSSÃO}

Constatou-se incidência de $A$. woglumi em todas as avaliações realizadas, sendo que o número médio total foi maior durante a estação chuvosa, principalmente nos meses de janeiro a março/2007, quando a praga atingiu seu ápice em aproximadamente 800 insetos/armadilha (Figuras 1 e 2). No entanto, em abril, mês com maior índice pluviométrico, houve queda brusca na infestação, sendo capturados, em média, 100 insetos/armadilha. Após esse período, até setembro, a ocorrência de picos populacionais oscilou numa periodicidade bimestral. Essa relação observada é uma possível explicação para a maior densidade populacional encontrada no período chuvoso, meses em que as plantas de citros estão em pleno desenvolvimento, emitindo novas brotações, local preferido para alimentação e oviposição.

Silva et al. (2011a) observaram índice elevado de plantas com presença de mosca-negra nos meses de baixa precipitação pluvial (abril e maio/2009), no Estado do Pará, mas também relacionaram o aumento populacional da praga ao maior crescimento vegetativo das plantas de citros, que ocorreu logo após um período intenso de chuvas, nos meses de janeiro, fevereiro e março/2009, e por essa fase ser a preferida para alimentação e oviposição.

Resultados divergentes também foram encontrados por Medeiros et al. (2009), observando que os maiores picos populacionais de $A$. woglumi foram no período de baixa precipitação, em áreas de produção de citros no Maranhão, avaliando somente número de posturas, ovos e ninfas do inseto presentes nas folhas de citros. É importante considerar a metodologia de coleta utilizada nesta pesquisa, uma vez que as chuvas intensas podem ter favorecido a dispersão dos adultos da mosca-negra e sua fixação nas armadilhas adesivas instaladas no pomar.

Constatou-se que houve correlação entre os fatores abióticos e a ocorrência de $A$. woglumi no pomar de citros em estudo (Tabela 1). Para a variável precipitação pluvial e número total de fêmeas + machos, a correlação foi positiva e significativa quando considerado o período completo de avalição (chuvoso + seco), e não significativa para o período chuvoso, que favoreceu a incidência da praga na cultura e sua provável dispersão e fixação nas armadilhas adesivas. Silva et al. (2011b) avaliaram a influência dos fatores abióticos na infestação natural de mosca-negra e constataram que a precipitação pluvial apresentou correlação não significativa $(r=$ $-0,464)$ e reduziu a presença do inseto nas plantas de citros, em Capitão Poço (PA).

Neste trabalho, a temperatura manteve-se praticamente constante e elevada ao longo do ano, condições normais para a região do Trópico Úmido. A correlação da variável temperatura foi significativa e apresentou, em todos os períodos, resultados negativos com a quantidade de insetos coletados, indicando que possíveis alterações na temperatura podem causar alterações na densidade populacional de A. woglumi (Tabela 1). De acordo com Gyeltshen et al. (2005), o desenvolvimento de A. spiniferus é muito influenciado pelo clima predominante de uma região, e pesquisas realizadas por Lopes et al. (2013) verificaram que, nas condições climáticas do Estado do Maranhão, A. woglumi apresenta-se como uma espécie multivoltina, completando seu ciclo de vida com $66,71 \pm 0,71$ dias, com possibilidade de ocorrer, em média, cinco gerações ao ano.

Silva et al. (2011b) também relataram que a temperatura se apresenta como elemento muito importante para o desenvolvimento da população de insetos e encontraram correlação significativa e positiva $(r=0,478)$ entre a temperatura média e o índice de plantas infestadas por mosca-negra em plantas cítricas, no Estado do Pará.

Para a variável umidade relativa, a correlação foi significativa e positiva no período completo (chuvoso + seco) para o número total de insetos coletados, verificando-se que altos índices de umidade relativa do ar favoreceram a incidência da praga (Tabela 1, Figura 1). Dentre os fatores abióticos que mais influenciam o desenvolvimento de $A$. woglumi, estão umidade relativa (70-80\%) e temperaturas na faixa de $20-34^{\circ} \mathrm{C}$ (EPPO, 1997). No Estado do Pará, este inseto encontra condições ideais de desenvolvimento, ou seja, temperatura entre 28 e $32{ }^{\circ} \mathrm{C}$ e umidade relativa de 70 a $80 \%$ (SILVA et al., 2001b), valores próximos aos encontrados nesta pesquisa.

Os semivariogramas obtidos caracterizaram agregação na distribuição espacial (Tabela 2 e Figura 3). Escolheu-se o modelo esférico para a obtenção dos variogramas com o intuito de descrever 
a estrutura espacial de $A$. woglumi nas estações chuvosa e seca para machos, fêmeas e total de insetos coletados, além do índice $\mathrm{k}$, que é outro indicador de ocorrência de distribuição espacial do organismo em estudo. Journel e Huijbregts (1978) determinaram que os valores obtidos para o índice $\mathrm{k}$ abaixo de 0,80 indicam que o fenômeno estudado é agregado, com forte relação entre as amostras, e, nesse trabalho, os índices encontrados variaram entre 0,14 e 0,25.

$O$ alcance da dependência espacial para adultos da mosca-negra, onde as amostras são consideradas espacialmente dependentes, durante a estação chuvosa para o número médio de machos, fêmeas e total de adultos, foi de 238,00 m, 209,60 $\mathrm{m}$ e 233,00 m, respectivamente (Figuras 3A, 3B e 3C). Portanto, a área de agregação da mosca-negra no pomar foi de $177.862 \mathrm{~m}^{2}$ para machos, 137.946 $\mathrm{m}^{2}$ para fêmeas e de $170.467 \mathrm{~m}^{2}$ para o número total de adultos.

$\mathrm{O}$ alcance da dependência espacial para a estação seca foi cerca de 3/4 menor do que para a estação chuvosa. Para o número de machos, o alcance foi de $54,40 \mathrm{~m}$, para fêmeas, igual a $55,70 \mathrm{~m}$, e para o total de adultos, de 55,90 m (Figuras 3D, 3E e 3F). Já a área de agregação de adultos da mosca-negra para a estação chuvosa foi de $9.292 \mathrm{~m}^{2}$, de $9.741 \mathrm{~m}^{2}$ e de $9.811 \mathrm{~m}^{2}$, para os números de machos, fêmeas e total de adultos, respectivamente.

Os mapas das densidades da população estimadas, interpoladas pelo método da krigagem, mostraram áreas de agregação dentro do pomar. $\mathrm{Na}$ estação chuvosa, os valores foram de 5,28 para machos (Figura 4A), de 4,54 para fêmeas (Figura 4B) e de 5,71 para a população total (Figura 4C). Notouse que as áreas de agregação obtidas foram muito próximas para os dois sexos bem como para o número total de adultos. Para a estação seca, as áreas com maior quantidade de insetos no pomar em estudo não se distinguiram muito das áreas de maior agregação na estação chuvosa, mas como o nível populacional foi menor nessa estação climática, a agregação também foi inferior. Para machos, na maior área de agregação, seriam encontrados, pelo menos, 3,90 insetos (Figura 5A), 3,62 fêmeas (Figura 5B) e 4,48 insetos, independentemente do sexo (Figura 5C).

Neste trabalho, pôde-se inferir que, com a área de agregação encontrada para a estação chuvosa, deve-se colocar, ao menos, uma armadilha a cada 17 ha, com a finalidade de se obter uma estimativa confiável das populações de mosca-negra num plano de amostragem. Já para a estação seca, é necessária uma armadilha por hectare para se obter a mesma estimativa. A caracterização da estrutura espacial de $A$. woglumi mostrou-se útil, por potencializar a importância de amostragens desse inseto durante o período seco, pois teve-se o menor alcance da dependência espacial encontrada nessa estação climática.

A distribuição do tipo agregada geralmente implica dependência espacial da variável número de espécimes por área, permitindo uma abordagem através da geoestatística e da utilização de ferramentas de agricultura de precisão, possibilitando, dessa maneira, o desenvolvimento de metodologia de amostragem da população dos insetos-praga. Pinho et al. (2008) verificaram que a distribuição espacial da mosca- negra ocorreu em agrupamentos com dependência espacial descrita pelo modelo esférico, formando reboleiras com alcances que variaram de 15,5 a $34 \mathrm{~m}$ em plantio de citros em sistema agroflorestal.

Os resultados encontrados neste trabalho indicaram que, na elaboração de um plano de amostragem, pode-se optar por qualquer um dos sexos ou o número total de insetos capturados devido às suas dependências espaciais serem muito próximas em ambas as estações e que o número de armadilhas a serem instaladas deverá ser maior na estação seca. 
TABELA 1 - Índices de correlação de Spearman entre número de adultos de Aleurocanthus woglumi capturados em armadilhas adesivas amarelas sustentadas em plantas de lima-ácida Tahiti e as variáveis climáticas precipitação pluvial $(\mathrm{mm})$, temperatura $\left({ }^{\circ} \mathrm{C}\right)$ e umidade relativa do ar (\%). São José de Ribamar - MA.

\begin{tabular}{|c|c|c|c|c|}
\hline \multirow{2}{*}{ Variável Climática } & \multirow{2}{*}{ Adultos } & \multicolumn{3}{|c|}{ Período } \\
\hline & & Chuvoso & Seco & Completo $^{1}$ \\
\hline Precipitação Pluvial & $(\mathrm{F}+\mathrm{M})^{2}$ & $0,4110^{\text {ns3 }}$ & $-0,0231^{\mathrm{ns}}$ & $0,5603 *$ \\
\hline Temperatura & $(\mathrm{F}+\mathrm{M})$ & $-0,6440^{*}$ & $-0,7455^{*}$ & $-0,7471 *$ \\
\hline Umidade Relativa & $(\mathrm{F}+\mathrm{M})$ & $0,3172^{\mathrm{ns}}$ & $0,3736^{\mathrm{ns}}$ & $0,5988 *$ \\
\hline
\end{tabular}

${ }^{1}$ o período completo de coletas refere-se aos períodos chuvoso e seco.

${ }^{2}$ total de fêmeas e machos de mosca-negra-dos-citros.

${ }^{3}$ significância dos coeficientes de correlação (r) de Spearman testada pelo teste t de Student, ao nível de 5\% de probabilidade.

TABELA 2 - Modelos de semivariograma para a distribuição espacial de adultos de Aleurocanthus woglumi, capturados em armadilhas adesivas amarelas sustentadas em plantas de lima-ácida Tahiti. São José de Ribamar-MA.

\begin{tabular}{|c|c|c|c|c|c|c|c|}
\hline \multicolumn{4}{|c|}{ Parâmetros do Semivariograma } & \multirow[b]{2}{*}{ Modelo } & \multirow[b]{2}{*}{$\mathbf{R}^{2}$} & \multirow{2}{*}{$\begin{array}{c}\text { Área de } \\
\text { agregação } \\
\left(\mathrm{m}^{2}\right)^{1}\end{array}$} & \multirow[b]{2}{*}{$\mathbf{K}^{2}$} \\
\hline & Efeito Pepita & Patamar & $a(m)$ & & & & \\
\hline \multicolumn{8}{|c|}{ Estação Chuvosa } \\
\hline Machos & 0,161 & 0,742 & 238,00 & Esférico & 0,85 & 177.862 & 0,21 \\
\hline Fêmeas & 0,139 & 0,960 & 209,60 & Esférico & 0,89 & 137.946 & 0,14 \\
\hline Total & 0,147 & 0,817 & 233,00 & Esférico & 0,89 & 170.467 & 0,18 \\
\hline \multicolumn{8}{|c|}{ Estação Seca } \\
\hline Machos & 0,188 & 0,748 & 54,40 & Esférico & 0,75 & 9.292 & 0,25 \\
\hline Fêmeas & 0,126 & 0,504 & 55,70 & Esférico & 0,72 & 9.741 & 0,25 \\
\hline Total & 0,132 & 0,610 & 55,90 & Esférico & 0,78 & 9.811 & 0,22 \\
\hline
\end{tabular}

${ }^{1}$ área de agregação $=\pi r^{2}$, em que $\pi=3,1415 \mathrm{er}=\mathrm{a}$.

2índice $\mathrm{k}=\mathrm{C}_{0} / \mathrm{C}_{0}+\mathrm{C}_{1}$.

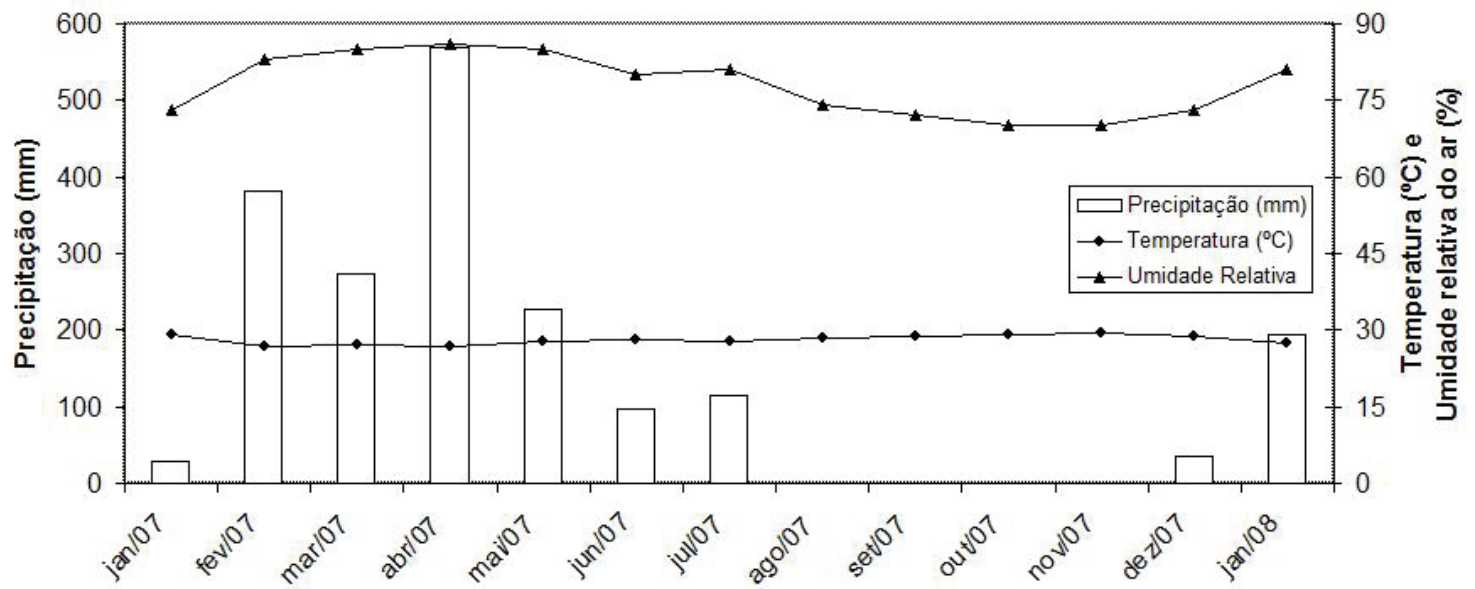

Meses de coleta

FIGURA 1- Temperatura média $\left({ }^{\circ} \mathrm{C}\right)$, umidade relativa do ar média (\%) e precipitação pluvial total (mm) mensais. São Luís-MA. 2007-2008. 


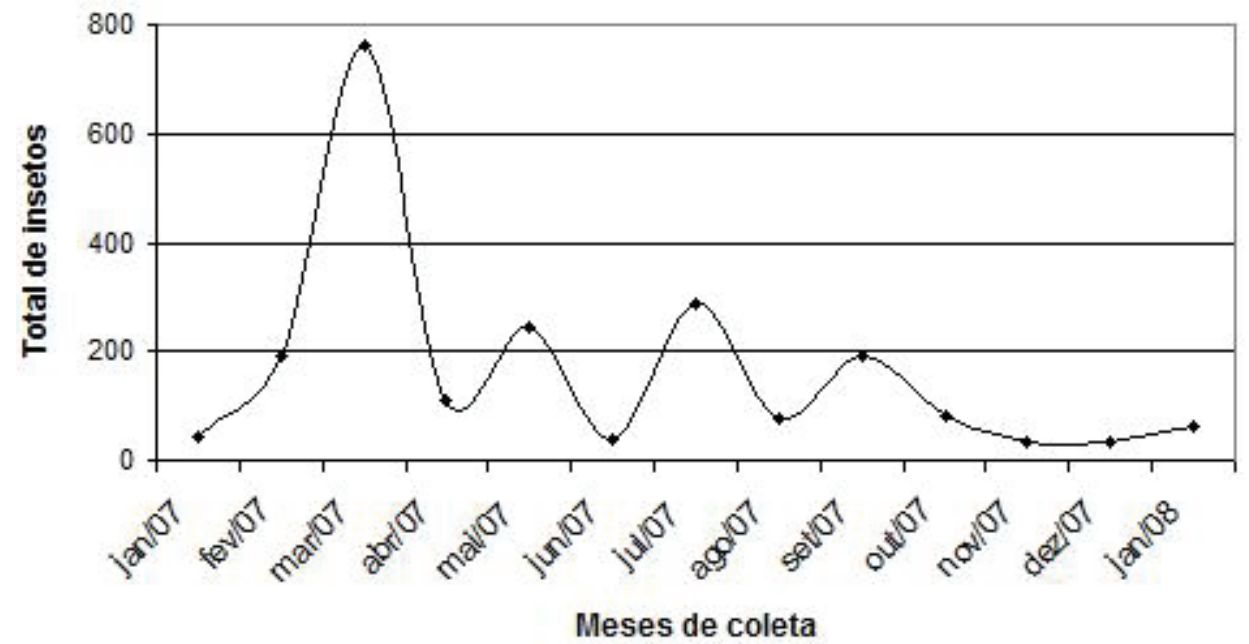

FIGURA 2 - Número médio de adultos de Aleurocanthus woglumi, capturados em armadilhas adesivas amarelas sustentadas em plantas de lima-ácida Tahiti. São José de Ribamar-MA.
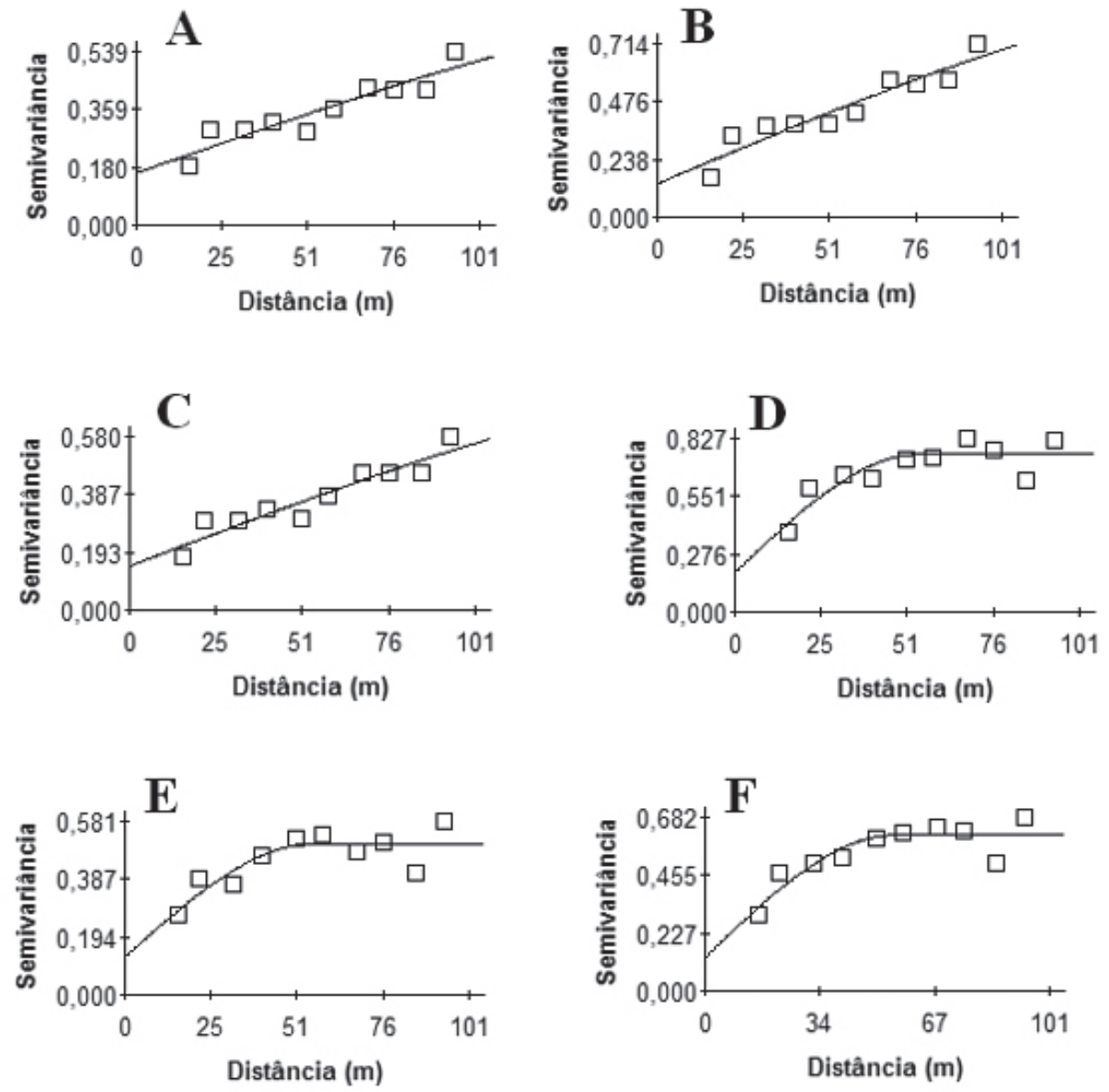

FIGURA 3 - Variogramas de adultos de Aleurocanthus woglumi capturados em armadilhas adesivas amarelas sustentadas em plantas de lima-ácida Tahiti em duas distintas estações climáticas: estação chuvosa $(\mathrm{A}=$ machos, $\mathrm{B}=$ fêmeas e $\mathrm{C}=$ total de adultos $)$; estação seca $(\mathrm{D}=$ machos, $\mathrm{E}=$ fêmeas e $\mathrm{F}=$ total de adultos). São José de Ribamar-MA. 

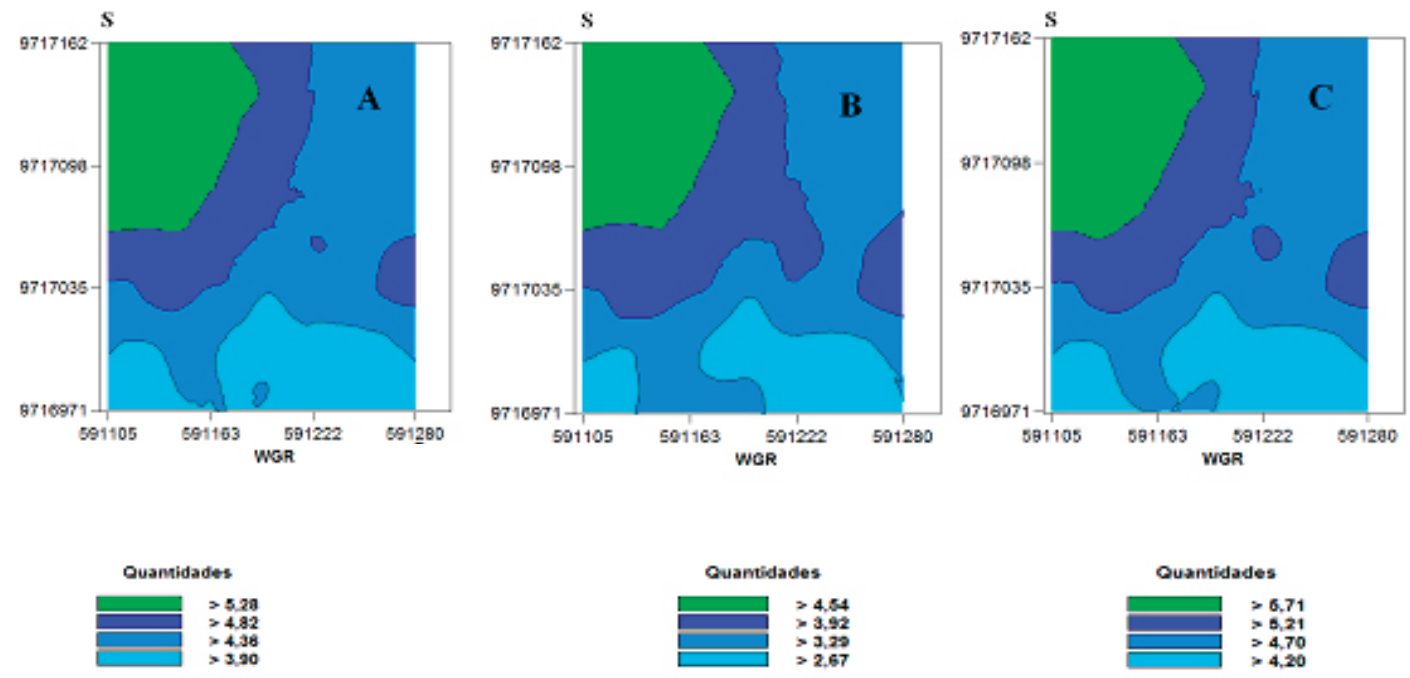

FIGURA 4 - Mapas demonstrando a distribuição espacial de adultos de Aleurocanthus woglumi capturados em armadilhas adesivas amarelas sustentadas em plantas de lima-ácida Tahiti na estação chuvosa $(\mathrm{A}=$ machos; $\mathrm{B}=$ fêmeas e $\mathrm{C}=$ total $)$. São José de Ribamar-MA.

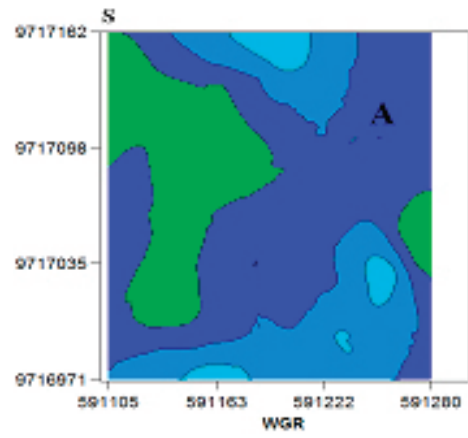

Quantidades

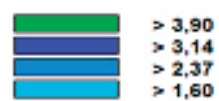

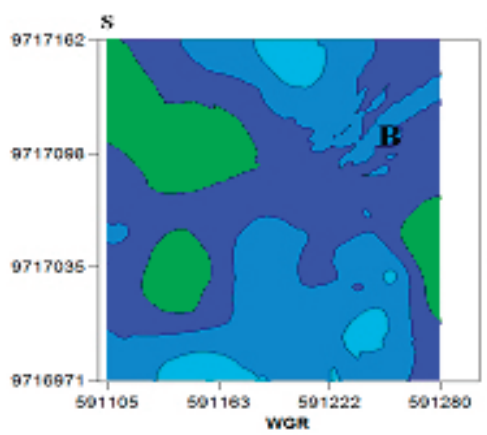

Quantidades

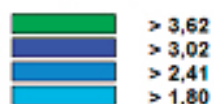

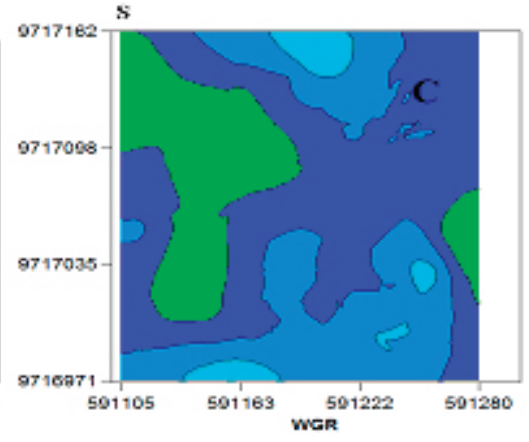

Quantidades

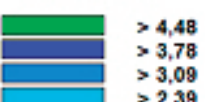

FIGURA 5 - Mapas demonstrando a distribuição espacial de adultos de Aleurocanthus woglumi capturados em armadilhas adesivas amarelas sustentadas em plantas de lima-ácida Tahiti na estação seca $(\mathrm{A}=$ machos; $\mathrm{B}=$ fêmeas e $\mathrm{C}=$ total). São José de Ribamar-MA.

\section{CONCLUSÃO}

O período chuvoso contribui para a elevação do nível populacional de Aleurocanthus woglumi.

A área de agregação de $A$. woglumi na estação chuvosa é maior que na estação seca, sendo que, para se obter uma estimativa confiável do número de adultos da mosca-negra-dos-citros, durante a estação chuvosa, é necessário instalar uma armadilha a cada 17 hectares e, durante a estação seca, uma armadilha a cada hectare.

\section{AGRADECIMENTOS}

À FAPEMA (Fundação de Amparo à Pesquisa e ao Desenvolvimento Tecnológico do Maranhão) pela concessão da bolsa de estudos para o primeiro autor. 


\section{REFERÊNCIAS}

ADAGRI - Agência de Defesa Agropecuária do Estado do Ceará. Ceará se prepara para erradicar a Mosca-Negra-Cítrus. 2010. Disponível em: $<\underline{\text { http:// }}$ www.ceara. gov.br/ index. php/ sala-de-imprensa/ noticias/1019-ceara-se-prepara-para-erradicar-a-

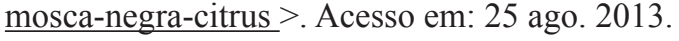

ADEAL-Agência de Defesa e Inspeção Agropecuária de Alagoas. Mosca-negra ameaça plantações de Alagoas. 2010. Disponível em: < http://www. defesa agropecuaria.al.gov.br/sala-de-imprensa/ noticias/2010/05/mosca-negra-ameaca plantacoesde-lagoas $/$ ? searchterm $=$ mosca $\% 20$ negra $>$ Acesso em: 25 ago. de 2013.

BLACKSHAW, R. P.; VERNON, R. S. Spatio temporal stability of two beetle populations in non-farmed habitats in an agricultural landscape. Journal of Applied Ecology, Oxford, v.43, n.4, p.680-689, 2006.

CORREIA, R. G.; LIMA, A. C. S.; FARIAS, P. R.S.; MACIEL, F. C. S.; SILVA, M. W.; SILVA, A. G. Primeiro registro da ocorrência de mosca-negrados-citros, Aleurocanthus woglumi Ashby, 1915 (Hemiptera: Aleyrodidae) em Roraima. Revista Agro@mbiente On-line, Boa Vista, v. 5, n. 3, p. 245-248, 2011.

EPPO/CABI - European and Mediterranean Plant Protection Organization. Aleurocanthus woglumi. In: SMITH, I. M.; MCNAMARA, D. G.; SCOTT, P. R.; HOLDERNESS, M. Quarantine pests for Europe. $2^{\text {nd }}$ ed. Wallingford: CAB International, 1997. p. 25-29.

FARIAS, P. R. S.; SÁNCHEZ-VILA, X.; BARBOSA, J. C.; VIEIRA, S.R.; FERRAZ, L. C. C. B. Geostatistical analysis of the spatial distribution of Rotylenchulus reniformis on cotton cultivated in crop rotation. Russian Journal of Nematology, Moscow, v. 10, n.1, p.1-9, 2002.

FERREIRA FILHO, P. J.; WILCKEN, C. F.; OLIVEIRA, N. C.; DAL POGETTO, M. H. F. A.; LIMA, A. C. V. Caracterização da estrutura espacial do psilídeo-da-concha Glycaspis blimblecomblei (Hemiptera: Psyllidae) e de seu parasitoide Psyllaephagus bliteus (Hymenoptera: Encyrtidae) em floresta de Eucalyptus camaldulensis. Boletín Sanidad Vegetal Plagas, Madrid, v. 34, n.1, p. 11-20, 2008.
FLETCHER, R. S.; EVERITT, J. H.; DAVIS, M. R.; ESCOBAR, D. E. Integrating airborne imagery and GIS technology to map and compare citrus blackfly infestations occurring in different years. HortTechnology, Alexandria, v. 14, n. 3, p. 398401, 2004.

GYELTSHEN, J.; HODGES, A.; HODGES, G. S. Orange spiny whitefly, Aleurocanthus spiniferus Quaintance (Insecta: Hemiptera: Aleyrodidae). EENY, Gainesville, n. 341, 2005. Disponível em: $<$ http://edis.ifas.ufl.edu/pdffiles/IN/IN61800.pdf $>$. Acesso em: 10 fev. 2010.

IBGE - Instituto Brasileiro de Geografia e Estatística. Levantamento sistemático da produção agrícola. 2014. Disponível em: <http://www.ibge.gov.br/ home/estatistica/indicadores/agropecuaria/lspa/ lspa 201307 4.shtm $>$ Acesso em: 25 ago. de 2014.

JOURnEL, A. G.; HUIJBREGTS, C. H. J. Mining geostatistics. London: Academic Press, 1978. 600p.

LEMOS, R. N. S.; SILVA, G. S.; ARAÚJO, J. R.G.; CHAGAS, E. F.; MOREIRA, A. A.; SOARES, A. T. M. Ocorrência de Aleurocanthus woglumi Ashby (Hemiptera: Aleyrodidae) no Maranhão. Neotropical Entomology, Londrina, v.35, n.4, p.558 - 559, 2006.

LOPES, E. B.; ALBUQUERQUE, I. C.; COSTA, F. R.; BORGES, J. A. M. Mosca-negra-dos-citros (Aleurocanthus woglumi Ashby) (Hemiptera: Aleyrodidae) chega à Paraíba. Lagoa Seca: EMEPA, 2009. 17p. 2009. (Relatório TécnicoFitossanitário).

LOPES, G. da S.; LEMOS, R. N. S.; ARAÚJO, J. R. G.; MARQUES, L. J. P.; VIEIRA, D. L. Preferência para oviposição e ciclo de vida de mosca-negra-doscitros Aleurocanthus woglumi Ashby em espécies frutíferas. Revista Brasileira de Fruticultura, Jaboticabal, v. 35, n. 3, p. 738-745, 2013.

MAPA - Ministério da Agricultura, Pecuária e Abastecimento. Lista de pragas quarentenárias presentes (A2). Disponivel em: $\leq$ http://extranet.agricultura.gov.br/sislegisconsulta/servlet/ VisualizarAnexo?id=14644>. Acesso em: 23 jun. 2014.

MATHERON, G. Principles of geostatistics. Economic Geology, Littleton, v.58, n.8, p.12461266, 1963. 
MATOS, C. A. O. de. Variabilidade espacial de Spodoptera frugiperda (J.E. Smith, 1797) (Lepidoptera: Noctuidae) no milho: métodos geoestatísticos e de estatística clássica. 2000. $72 \mathrm{f}$. Dissertação (Mestrado em Agronomia) - Faculdade de Ciências Agronômicas, Universidade Estadual Paulista, Botucatu, 2000.

MARUYAMA, W. I.; BARBOSA, J. C.; TOSCANO, L. C. Distribuição espacial de Oncometopia facialis (Signoret) (Hemiptera: Cicadellidae) em pomar cítrico. Neotropical Entomology, Londrina, v. 35, n.1, p.93-100, 2006.

MEAGHER, R. L.; FRENCH, J. V.; ESAU, K. L. Monitoring and biological control of citrus blackfly in south Texas. Subtropical Plant Science, Weslaco, v. 44, p.19-24, 1991.

MEDEIROS, F. R.; LEMOS, R. N. S.; OTTATI, A. L. T.; ARAÚJO, J. R. G.; MACHADO, K. K. G. Dinâmica populacional da mosca-negra-doscitros Aleurocanthus woglumi ashby (Hemiptera: Aleyrodidae) em Citrus spp. no município de São Luís - MA. Revista Brasileira de Fruticultura, Jaboticabal, v.31, n.4, p.1016-1021, 2009.

MONTEIRO, B. S.; RODRIGUES, K.C.V.; SILVA, A. G.; BARROS, R. Ocorrência da mosca-negra-doscitros (Aleurocanthus woglumi ASHBY) (Hemiptera: Aleyrodidae) em Pernambuco. Revista Caatinga, Mossoró, v.25, n.2, p.173-176, 2012.

NGUYEN, R.; BRAZZEL, J. R.; POUCHER, C. Population density of the citrus blackfly, Aleurocanthus woglumi Asbhy (Homoptera: Aleyrodidae) an its parasites in urban Florida in 1979-1981. Environmental Entomology, Maryland, v.12, n.3, p.878-884, 1983.

PINHO, R. C.; FARIAS, P. R. S.; BOIÇA JÚNIOR, A. L.; SILVA, A. G.; CORREIA, R. G.; PONTE, N. H. T. Caracterização espacial da mosca-negra (Aleurocanthus woglumi Ashby) em pomar de citros, em sistema de plantio agroflorestal na Amazônia Oriental, utilizando a metodologia geoestatística. In: XXII CONGRESSO BRASILEIRO DE ENTOMOLOGIA, 22., 2008, Uberlândia. Anais... Uberlândia: SEB, 2008.

RAGA, A.; COSTA, V. A. Mosca-negra-dos-citros. São Paulo: Agência Paulista de Tecnologia dos Agronegócios/ Instituto Biológico, 2008. p.1-9. (Documento Técnico, 001). Disponível em: $<$ http:// www.biologico.sp.gov.br/docs/dt/mosca_negra.pdf $>$. Acesso em: 26 mar. 2012.
RONCHI-TELES, B.; PENA, M. R.; SILVA, N. M. Observações sobre a ocorrência de Mosca-Negrados-Citros, Aleurocanthus woglumi Ashby, 1915 (Hemiptera: Aleyrodidae) no Estado do Amazonas. Acta Amazônica, Manaus, v.39, n.1, p.241-244, 2009.

SAS Institute. System. Version 8.2. Cary, 2001. 6 CD-ROM. Windows 98.

SILVA, S. X. de B.; SOARES, A. C. F.; MAIA, W. J. M. S.; LOBO, C. G. B.; RODRIGUES, D.; FROIS, R.J. Mosca-negra-dos-citros (Aleurocanthus woglumi Ashby) na Bahia: Detecção e medidas de controle. In: REUNIÃO REGIONAL DA SBPC NO RECÔNCAVO DA BAHIA, 2010, Cruz das Almas. Anais eletrônicos... Cruz das Almas: UFRB, 2010. Disponível em: <http://www.sbpcnet.org.br/livro/ reconcavo/listatodos.htm> . Acesso em: 15 ago. 2013.

SILVA, A. B.; GUEIREDO, H. B de; MELO, M. M. A Mosca Negra dos Citros (Aleurocanthus woglumi Ashby): Todo cuidado é pouco! Proteja seu pomar. Não deixe esta praga entrar. Belém: Ministério da Agricultura Pecuária e Abastecimento /Secretaria Executiva de Agricultura, 2001.

SILVA, A. G.; BOIÇA JUNIOR, A. L.; FARIAS, P. R. S.; BARBOSA, J. C. Infestação da mosca-negrados-citros (Aleurocanthus woglumi Ashby) em pomares de citros em sistema de plantio convencional e agroflorestal. Revista Brasileira de Fruticultura, Jaboticabal, v.33, n.1, p.53-60, 2011a.

SILVA, A. G.; BOIÇA JUNIOR, A. L.; FARIAS, P. R. S. Influência da temperatura e precipitação na infestação de mosca-negra-dos-citros (Aleurocanthus woglumi) em plantio de citros. Revista Nucleus, Ituverava, v.8, n.1, p. 385-392, 2011 b.

TAYLOR, L. R. Assessing and interpreting the spatial distributions of insect populations. Annual Review of Entomology, Palo Alto, v. 29, p. 321-357, 1984.

TEIXEIRA, M. B. R. Comparação entre estimadores de semivariância. 2013. 122p. Dissertação (Mestrado em Estatística e Experimentação Agropecuária) Universidade Federal de Lavras, 2013. 